\title{
Memory lane
}

\author{
Robert Dennard and 50 years of dynamic random access memory.
}

n 9 November 1966, while sitting in the living room of his home in

Westchester County, New York, Robert Dennard began to formulate an idea that would help deliver a revolution in computer technology. The idea was to create a memory cell from a single capacitor and transistor: a bit of data would be stored in the capacitor (its charged and uncharged states providing the necessary ' 1 ' and ' 0 ') and the transistor would be used to write the information to the capacitor. Dennard, who worked at IBM's Thomas J. Watson Research Center, continued to consider and refine the idea over the coming months, before finally realizing that the transistor could be used to read, as well as write, the charge stored in the capacitor. One-transistor dynamic random access memory - DRAM was born.

The invention of DRAM is a story that Dennard recounts in our Reverse Engineering column in this issue of Nature Electronics. It is a story driven by a search for simplicity: to move away from the bulky magnetic-core random access memory that was common at the time and to refine previous transistor-based memory cell designs. In 1967, IBM filed for a patent on the technology and it was issued on 4 June 1968 - 50 years ago this month.

In 1970, Intel, which had been formed by Robert Noyce and Gordon Moore only two years previous, launched the first commercial DRAM chip - the Intel 1103 (pictured). The chip had 1,024 bits of memory and used a three-transistor memory cell. It was priced at US\$21, which worked out at about 2 cents a bit ${ }^{1}$. For Intel, the chip was a timely success. By 1972, it was the biggest selling semiconductor memory in the world and accounted for almost all of the company's revenue ${ }^{2}$, which had by then jumped to US\$23 million ${ }^{1}$. Moore would also later describe, in IEEE Spectrum magazine $^{3}$, the Intel 1103 as perhaps his favourite chip, explaining: "It was the chip that really got Intel over the hump to profitability." (In the same article, Morris Chang, the founder of TSMC, would also incidentally choose the Intel 1103 as his favourite chip.) By the mid-1970s,

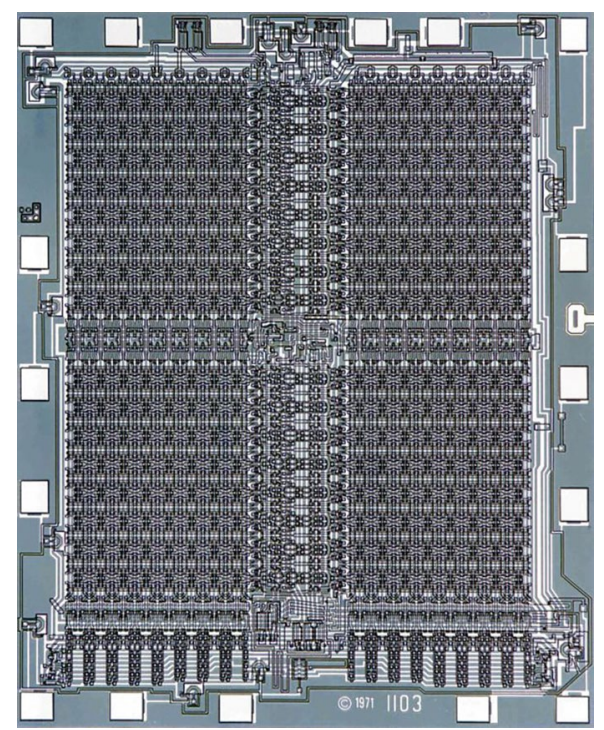

Photograph of the Intel 1103 DRAM die. The Intel 1103 had 1,024 bits of memory and was the first commercially available DRAM chip.Credit: image provided courtesy of Intel Corporation

a variety of manufacturers had introduced four-kilobit DRAM chips. These included the Mostek MK4096, which was invented by Mostek cofounder Robert Proebsting and offered space-saving 16-pin packaging that would become an industry standard ${ }^{4}$.

In DRAM, the data is only stored temporarily, as the charge in the capacitors leaks over time, and the memory cells have to be refreshed regularly. Hence it is known as a 'dynamic' memory and is in contrast to static RAM, which does not require the data to be refreshed. Nevertheless, the role of DRAM in the development of microelectronics was profound. As Dennard notes: "The simplicity and low power consumption of DRAM changed the computing industry. It allowed RAM to become very dense and inexpensive. As a result, mainframe computers could be equipped with relatively fast RAM to act as a buffer to the increasing amount of data stored on disk drives. This vastly sped up the process of accessing and using stored information. Just as important, DRAM was combined with microprocessors to make personal computers possible."

For Dennard, there was to be a second act to his role in the history of microelectronics. In 1972, in a talk at the International Electron Devices Meeting in Washington, DC, he introduced scaling principles for metal-oxide-semiconductor field-effect transistors (MOSFETs) ${ }^{5}$, which would later become known as Dennard scaling. However, it was his subsequent 1974 paper - co-authored with IBM colleagues Fritz Gaensslen, Hwa-Nien Yu, V. Leo Rideout, Ernest Bassous and Andre LeBlanc that attracted wider attention and is the document most closely associated with Dennard scaling 6 .

The paper provides scaling rules for achieving improvements in transistor density, switching speed and power dissipation. It, in fact, also reports scaling results for interconnect lines, though that section is less often discussed. The work shows, in particular, that the power density of the transistors remains roughly the same as they become smaller, and thus as transistors shrink so does their required voltage and current. Together with Moore's law ${ }^{7,8}$, it provided a broad blueprint for consistent development in the microelectronics industry, which persisted for around 30 years ${ }^{9}$.

Published online: 13 June 2018 https://doi.org/10.1038/s41928-018-0098-9

References

1. Thackray, A., Brock, D. C. \& Jones, R. Moore's Law: The Life of Gordon Moore, Silicon Valley's Quiet Revolutionary (Basic Books, New York, 2015).

2. A Revolution in Progress: A History of Intel to Date (Intel Corporation, 1984); https://go.nature.com/2JldLrK

3. Technology luminaries describe the microchips that changed the world - and their lives. IEEE Spectrum (1 May 2009); https://go.nature.com/2ssB88c

4. Chip hall of fame: Mostek MK4096 4-kilobit DRAM. IEEE Spectrum (30 June 2017); https://go.nature.com/2J8D1hM

5. Dennard, R. H., Gaensslen, F. H., Kuhn, L. \& Yu, H. N. IEDM Tech. Digest 168-170 (1972).

6. Dennard, R. H. et al. IEEE J. Solid-State Circuits 9 , 256-268 (1974)

7. Moore, G. E. Electronics 38, 114-117 (1965).

8. Moore, G. E. IEDM Tech. Digest 21, 11-13 (1975).

9. Bohr, M. IEEE Solid-State Circuits Society Newsletter 12, $11-13$ (2007) 